\title{
Risk factors for unsuccessful lumbar puncture in children
}

\author{
C Procter, ${ }^{1,2}$ MBBS, FCPaed (SA); H Buys, ${ }^{1,2}$ MB ChB, FCPaed (SA); H Carrara, ${ }^{3}$ MPH; J Thomas, ${ }^{1,4}$ MB ChB, FFA \\ ${ }^{1}$ Red Cross War Memorial Children's Hospital, Cape Town, South Africa \\ ${ }^{2}$ Department of Paediatrics and Child Health, Faculty of Health Sciences, University of Cape Town, South Africa \\ ${ }^{3}$ School of Public Health and Family Medicine, Faculty of Health Sciences, University of Cape Town, South Africa \\ ${ }^{4}$ Department of Anaesthesia, Faculty of Health Sciences, University of Cape Town, South Africa
}

Corresponding author: C Procter (clairemprocter@gmail.com)

\begin{abstract}
Background. This descriptive study provides the first information on an association between the use of sedation and a reduction in the prevalence of unsuccessful lumbar puncture (LP) in African children of all races.

Objective. Our hypothesis was that children who do not receive any procedural sedation are more likely to have unsuccessful LPs.

Methods. A cross-sectional observational study examined LPs performed from February to April 2013, including details of the procedure, sedation or analgesia used, and techniques. The setting was the Medical Emergency Unit at Red Cross War Memorial Children's Hospital, Cape Town, South Africa, and the participants all children aged 0 - 13 years who had an LP in the unit during the time period.

Results. Of 350 children, $62.9 \%$ were $<12$ months of age, the median age being 4.8 months (interquartile range $1.5-21.7$ ). The prevalence of unsuccessful (traumatic or dry) LP was 32.3\% (113/350). Sedation was used in 107 children (30.6\%) and was associated with a reduction in the likelihood of unsuccessful LP ( $p=0.002$; risk ratio (RR) 0.5 ( $95 \%$ confidence interval (CI) $0.34-0.78)$ ) except in those $<3$ months of age, where sedation did not significantly reduce the likelihood ( $p=0.56$; RR 1.20 ( $95 \%$ CI $0.66-2.18)$ ).

Conclusions. Unsuccessful LP was common. Sedation was not routinely used, but the results suggest that it may be associated with a reduction in the rate of unsuccessful LP. Unsuccessful LP may lead to diagnostic uncertainty, prolonged hospitalisation and unnecessary antibiotic use. Whether a procedural sedation protocol would reduce the rate of unsuccessful LP requires further study.
\end{abstract}

S Afr Med J 2016;106(12):1230-1235. DOI:10.7196/SAMJ.2016.v106i12.10703

Bacterial meningitis is a serious disease affecting adults and children, which results in an estimated 180000 deaths of children aged 1 month - 5 years per year worldwide. ${ }^{[1]}$ The rapid treatment and diagnosis of meningitis is crucial to reducing morbidity and mortality. ${ }^{[2]}$ Lumbar punctures (LPs) are commonly performed in paediatric emergency medicine departments to diagnose or exclude meningitis. LPs are considered to be traumatic when red blood cells are introduced into the CSF as a result of needle trauma to the epidural venous plexus lying against the vertebral bodies. ${ }^{[3]}$ Studies have reported the incidence of traumatic LPs to be between $10 \%$ and $30 \%{ }^{[3]}$ using cut-offs of between 400 (which is the visual threshold) and 10000 red blood cells (RBCs)/ $\mu \mathrm{L}$. The diagnosis or exclusion of meningitis may be confounded by the presence of any RBCs in the cerebrospinal fluid (CSF), so we chose to use the lowest cut-off of 400 $\mathrm{RBCs} / \mu \mathrm{L}$ in our study. The blood in the CSF sample also results in increased white blood cell (WBC) counts. The WBC count in the CSF is often used to determine whether a patient has meningitis or not. In the era of prehospital antibiotic use, the culture of the CSF cannot be relied upon so WBC counts are used.

Several studies have examined risk factors for unsuccessful or traumatic LP. None examined the influence of sedation on the success of LP. The conclusions with regard to other risk factors were not consistent. Most have agreed that younger patient age ( $<1$ year), not using local anaesthetic, late removal of the stylet and increased patient movement worsen outcomes. ${ }^{[3-5]}$ The influence of age $<3$ months and physician experience is less clear.

Unsuccessful LPs are traumatic for the child and parents, especially when repeat procedures are needed. They also cause diagnostic uncertainty which may prolong hospital stay and result in unnecessary antibiotic treatment with cephalosporins, use of which puts the child at risk of acquiring extended-spectrum beta-lactamase-producing organisms. ${ }^{[6]}$
Approximately 3500 LPs are done per year at Red Cross War Memorial Children's Hospital (RCWMCH), Cape Town, South Africa (SA), and in 2009 the proportion of traumatic LPs was found to be $17 \%$ of samples sent to the laboratory ( $\mathrm{R}$ Muloiwa, personal communication, 2014). That study did not include LPs when no samples were sent to the laboratory, so the number of unsuccessful LPs is unknown.

\section{Objective}

The high incidence of unsuccessful LPs observed, with the potential adverse consequences of increased hospital stays, led us to undertake an audit of current practice in order to identify what factors may be important in reducing traumatic LPs in our setting. We aimed to identify any modifiable risk factors, including whether the use of sedation is important, as there is currently no evidence for or against this. Using the results of the study we could then introduce a protocol for LPs aimed at reducing the number of unsuccessful LPs and improving the diagnostic yield. It was hoped that this would reduce unnecessary treatment and stay in hospital. There have been no previous studies from Africa addressing the issue of unsuccessful LPs or the use of procedural sedation in children undergoing LP.

Our hypothesis was that children who do not have any procedural sedation are more likely to have an unsuccessful LP.

\section{Methods}

This was a cross-sectional observational study. All children who had an LP in the Medical Emergency Unit (MEU) at RCWMCH over a 3-month period were included. Children were excluded if the LP was done in other hospitals or other wards (owing to concerns of bias, as procedures may be different for non-emergency LPs) or if they had 
congenital abnormalities of the spine, a history of a bleeding disorder, evidence of vasculitis or cellulitis over the lumbar spine, or a final diagnosis of subarachnoid bleeding from traumatic brain injury or herpes simplex infection on folder review.

The study participants were identified each day by examining the ward registers of the MEU. Children with diagnoses suggesting that they would have had an LP, such as neonatal sepsis, meningitis or fever of unknown origin, were identified and the folders were obtained to see if they had had an attempted LP. The doctors who performed the procedures were then asked to complete a questionnaire detailing the procedure (Appendix 1). The CSF results were obtained from the laboratory. The questionnaires were also available in the department for doctors to complete after any LP they performed. The data capture sheet included patient factors (age, sex), the experience of the person performing the LP and the person holding the child, the needle used, timing of stylet removal, the presence of a family member, any sedation and analgesia used, a rating of the amount of movement of the child, and the result of the procedure. All data were kept anonymous and confidential.

Ethics approval was obtained from the Faculty of Health Sciences Human Research Ethics Committee of the University of Cape Town (HREC ref. no. 173/2013) and from the RCWMCH hospital administration (ref. no. 694/13).

Given an estimated prevalence of unsuccessful LPs of $30 \%$, the study was powered to detect a $50 \%$ reduction in the proportion of unsuccessful LPs with the use of sedation with an alpha level of 5\%. This was chosen as being a clinically significant reduction. Power analysis demonstrated that 300 subjects would be enrolled for $80 \%$ power. The data collected were then analysed using Stata version 13 (StataCorp, USA). Conventional descriptive methods (means and standard deviations, medians with interquartile ranges (IQRs) or proportions) were used to describe and characterise the study population, e.g. gender, age in months, and number of traumatic LPs. The prevalence of unsuccessful LPs in our study was determined. The association between unsuccessful and successful LPs and categorical predictor variables was estimated using generalised linear regression modelling. Owing to the high prevalence $(>10 \%)$ of the outcome these are reported as risk ratios (RRs) and their respective 95\% confidence intervals (CIs). ${ }^{[7]}$

\section{Definitions}

Traumatic LP: $>400 \mathrm{RBCs} / \mu \mathrm{L}$, which is the visual threshold. ${ }^{[8]}$

Unsuccessful LP: Traumatic LP or dry, i.e. no CSF obtained. ${ }^{[3,8]}$

Early stylet removal: Removal of the stylet after passage through the epidermal and subcutaneous tissues rather than in the subarachnoid space..$^{[3,5]}$

Number of attempts: Number of times the needle penetrated the skin (redirecting the needle without exiting the skin is a single attempt).

Experienced holder: Qualified member of staff rather than a student.

Experienced physician: Performed $>50$ previous LPs.

\section{Results}

During the study period, 356 LPs were identified. Repeat procedures following an unsuccessful LP were not included. Data forms were not completed for six of the procedures, so the analysis was done on 350 procedures. Of these patients, $142(40.6 \%)$ were female and $208(59.4 \%)$ male. Of the procedures performed, 220 (62.9\%) were on infants $<12$ months of age and $158(45.1 \%)$ were on infants
$<3$ months of age. The age range was 3 days -12.6 years, with a median age of 4.8 months (IQR 1.5 - 21.7).

Of the 350 LPs, 113 were unsuccessful with either dry or bloody taps, giving a prevalence of $32.3 \%$ ( $95 \%$ CI $0.27-0.37$ ). Of these, four were dry taps and the rest were frank blood or bloodstained CSF $(>400 \mathrm{RBCs} / \mu \mathrm{L})$.

Sedation was used in $107 / 349$ cases (30.7\%). In one case it was not clear whether sedation had been used or not. Sedation was associated with a reduction in the likelihood of an unsuccessful LP $(p=0.002$; RR 0.5 (95\% CI $0.34-0.78)$ ). Unsedated patients had a rate of unsuccessful taps of $38.0 \%$ compared with $19.6 \%$ for patients who were sedated, showing a $51.6 \%$ reduction in unsuccessful procedures when sedation was used. Almost all patients who were sedated were given ketamine $(93 / 107,86.9 \%)$, either as a single agent or in combination with midazolam (7 patients). Two patients were given midazolam only. Chloral hydrate was used for 18 patients, alone (12 patients) or in combination with ketamine (6 patients). These numbers were too small to allow statistical comparisons between different methods of sedation.

The use of sedation was strongly correlated with age. Very few infants were given sedation, only $12 / 158(7.6 \%)$ of those aged $<3$ months receiving sedation, while $95 / 191(49.7 \%)$ of those aged $\geq 3$ months received it $(p<0.001$; RR 2.2 (95\% CI $1.89-2.65)$ ). Further analysis showed that sedation was not associated with a reduction in the proportion of unsuccessful LPs in infants aged $<3$ months ( $p=0.56$; RR $1.2(95 \%$ CI $0.66-2.12)$ ), but the number who received sedation in this age group was very small.

Forty-six of the 107 children who were sedated (43.0\%) were said not to be moving at all. Without sedation, 56/242 (23.1\%) were said not to be moving at all. Lack of sedation was associated with a significant increase in the rating of how much the child was moving (from 'not at all' to 'a little but did not affect the procedure' to 'a lot - difficult procedure') ( $p=0.001$; RR 0.65 (95\% CI 0.50 - 0.86)), and increasing movement of the child was associated with a higher proportion of unsuccessful LPs $(p<0.001$; RR 4.08 (95\% CI 2.54 6.56)).

The data were also analysed to identify other potential factors that may have influenced the outcome of the LP. These are summarised in Table 2.

The strongest predictor of unsuccessful LP was age $<12$ months. Analysis of patients by age showed that the proportion of unsuccessful LPs increased significantly in infants aged $<12$ months (Table 3 ), but there was no further increase in those aged $<3$ months. ${ }^{[5]}$

No significant difference in the rate of unsuccessful LP was found overall when a eutectic mixture of local anaesthetic (EMLA) was or was not used as a local anaesthetic cream for the LP. However, among those patients who received no sedation $(n=242)$, the proportion of unsuccessful LPs was $29.4 \%$ (15/51) when EMLA was used, whereas those without any analgesia or sedation had a proportion of $40.3 \%$ (77/191). This suggests that the proportion of unsuccessful LPs in unsedated patients was reduced when EMLA cream was used, but the numbers using EMLA were small and the reduction was not statistically significant ( $p=0.15$; RR 0.73 (95\% CI $0.46-1.16)$ ).

Of practitioners who used a needle with a stylet, only $15.4 \%$ (45/292) removed the stylet early, the rest removing it once in the subarachnoid space. In three cases the timing of the removal of the stylet was unknown. When the stylet was removed early the proportion of unsuccessful LPs was $46.7 \%$ (21/45), compared with $30.4 \%$ (75/247) with later removal. The number of practitioners who removed the stylet early was small, but this practice was associated 
with an increase in the rate of unsuccessful LPs ( $p=0.02$, RR $1.54(95 \%$ CI $1.07-2.22)$ ).

It was noted that of the procedures where only one attempt was made, $19.0 \%(32 / 168)$ were unsuccessful. When more than one attempt was made the proportion increased to $51.9 \%$ (54/104), showing that repeated passes of the needle resulted in an increased risk of unsuccessful LP ( $p<0.001$; RR 0.95 (95\% CI $0.70-1.31))$. Use of sedation was not significantly associated with a reduction in the number of procedures requiring more than one attempt $(p=0.47$, RR 0.87 (95\% CI 0.59 - 1.27)).

No significant difference was found in the proportion of unsuccessful LPs with increased experience of the physician or holder, presence of a family member, or LPs done during the day as opposed to the night.

\section{Discussion}

This observational study provides information on the use of sedation as a risk factor for unsuccessful LP in African children of all races. We are also able to give information on its prevalence in our department. The lower rate of traumatic LP observed by Howard et al. ${ }^{[4]}$ in their study of patients with leukaemia, almost all of whom were sedated, would suggest that sedation is beneficial in reducing traumatic LPs, but this is the first study to compare sedated with non-sedated patients. The $50 \%$ reduction in the incidence of unsuccessful LPs when sedation was used in this study leads us to recommend that protocolised sedation be used wherever possible for LP. Sedation has previously been shown to be safe when guidelines are followed, ${ }^{[9]}$ and the trauma and pain experienced by the child should be reduced if sedation is used.

Table 1. Effect of sedation according to age

\begin{tabular}{|c|c|c|c|c|c|}
\hline \multirow[b]{2}{*}{ Age group } & \multirow[b]{2}{*}{ Sedation } & \multirow[b]{2}{*}{$N$} & \multicolumn{2}{|c|}{ Prevalence of unsuccessful } & \multirow[b]{2}{*}{ RR $(95 \%$ CI $)$} \\
\hline & & & LP, $n(\%)$ & $p$-value & \\
\hline \multirow[t]{2}{*}{$<3$ months } & No & 146 & $61(41.7)$ & & 1.0 (ref) \\
\hline & Yes & 12 & $6(50.0)$ & 0.56 & $1.20(0.66-2.12)$ \\
\hline \multirow[t]{2}{*}{$\geq 3$ months } & No & 96 & $31(32.3)$ & & 1.0 (ref) \\
\hline & Yes & 95 & $15(15.8)$ & 0.01 & $0.5(0.28-0.85)$ \\
\hline
\end{tabular}

Table 2. Predictors of unsuccessful LP estimated by prevalence and RRs (and associated 95\% CIs)

\begin{tabular}{|c|c|c|c|c|c|}
\hline Variable & & $N$ & $\begin{array}{l}\text { Unsuccessful LP, } \\
n(\%)\end{array}$ & $p$-value & RR $(95 \%$ CI $)$ \\
\hline \multirow[t]{2}{*}{ Age } & $<12$ months & 220 & $95(43.2)$ & & 1.0 (ref) \\
\hline & $\geq 12$ months & 130 & $18(13.8)$ & $<0.001$ & $0.34(0.22-0.53)$ \\
\hline \multirow[t]{2}{*}{ EMLA } & No & 277 & $91(32.9)$ & & $1.0(\mathrm{ref})$ \\
\hline & Yes & 73 & $20(27.4)$ & 0.47 & $0.86(0.58-1.29)$ \\
\hline \multirow[t]{2}{*}{ Sedation } & No & 242 & $92(38.2)$ & & $1.0(\mathrm{ref})$ \\
\hline & Yes & 107 & $20(18.7)$ & 2 & $0.50(0.34-0.78)$ \\
\hline \multirow[t]{2}{*}{ Early stylet removal } & No & 247 & $74(30.0)$ & & $1.0(\mathrm{ref})$ \\
\hline & Yes & 45 & $21(46.7)$ & 0.02 & $1.54(1.07-2.22)$ \\
\hline \multirow[t]{2}{*}{ Experience of holder } & Student & 32 & $10(31.3)$ & & $1.0(\mathrm{ref})$ \\
\hline & Staff & 318 & $102(32.1)$ & 0.90 & $1.04(0.60-1.78)$ \\
\hline \multirow[t]{3}{*}{ Movement } & None & 102 & $17(16.7)$ & & $1.0(\mathrm{ref})$ \\
\hline & Some & 198 & $61(30.8)$ & 0.01 & $1.88(1.16-3.04)$ \\
\hline & A lot & 50 & $34(68.0)$ & $<0.001$ & $4.08(2.54-6.56)$ \\
\hline Experience of physician & $\leq 50$ & 49 & $18(36.7)$ & & $1.0(\mathrm{ref})$ \\
\hline (LPs performed) & $>50$ & 301 & $96(31.9)$ & 0.46 & $0.86(0.58-1.29)$ \\
\hline \multirow[t]{2}{*}{ Parental presence } & No & 322 & $103(32.0)$ & & $1.0(\mathrm{ref})$ \\
\hline & Yes & 28 & $10(35.7)$ & 0.68 & $1.12(0.66-1.88)$ \\
\hline
\end{tabular}

EMLA $=$ eutectic mixture of local anaesthetic.

Table 3. Number of procedures for each age range and rates of unsuccessful LP

\begin{tabular}{|c|c|c|c|c|}
\hline Age & Procedures, $N$ & $\%$ of total & $\begin{array}{l}\text { Rate of unsuccessful LP, } \\
n(\%)\end{array}$ & RR (95\% CI) \\
\hline 1 - 28 days (neonate) & 53 & 15.1 & $23(43.4)$ & 1 (ref) \\
\hline 1 - 3 months & 105 & 30.0 & $44(41.9)$ & $0.97(0.67-1.40)$ \\
\hline$\geq 3$ - 12 months & 62 & 17.7 & $28(45.2)$ & $1.05(0.70-1.56)$ \\
\hline$\geq 1-5$ years & 85 & 24.3 & $13(15.3)$ & $0.37(0.22-0.64)$ \\
\hline$\geq 5$ - 13 years & 45 & 12.9 & $5(11.1)$ & $0.26(0.10-0.68$ \\
\hline Total & 350 & 100.0 & $113(32.3)$ & \\
\hline
\end{tabular}


The majority (99.1\%) of LPs in our study were done to diagnose or exclude meningitis. Accuracy of diagnosis is crucial for the patient and for the doctor to determine the correct treatment. CSF culture results cannot be relied upon in an era of extensive prehospital antibiotic use due to implementation of the Integrated Management of Childhood Illness (IMCI) guidelines in SA. This means that the WBC count often has to be used for diagnosis of meningitis. Of 350 LPs in this study, only seven had positive CSF cultures. The diagnosis or exclusion of meningitis may be difficult if the LP is unsuccessful. Various correction methods have been proposed to help interpret traumatic LPs, but none of them can be certain to identify all cases of bacterial or tuberculous meningitis. ${ }^{[3]}$ If blood alone or no CSF is obtained, the LP cannot be used for diagnosis. The 32.3\% prevalence of unsuccessful LPs means that the diagnosis may not be clear in one-third of our patients undergoing LP. This may result in repeated procedures, unnecessary treatment, increased costs to the hospital, and increased financial and/ or emotional costs for the patient. The rate of $32.3 \%$ is consistent with previous studies, although it is one of the higher rates observed.

The factors examined in this study that increased the rate of unsuccessful LP were age $<12$ months, not using sedation, late removal of the stylet and increased movement of the patient. The finding of age as a predictor of unsuccessful LP is consistent with previous studies. ${ }^{[4,5,10]}$ In one previous study ${ }^{[5]}$ an increased incidence in patients aged $<3$ months compared with those aged $3-12$ months was also seen, but this was not observed in our study.

Previous studies have shown the benefit to the patient of using local anaesthetic in reducing the pain associated with LP. ${ }^{[11,12]}$ They have also shown that local anaesthetic increases the success of LP. ${ }^{[3,5,13]}$ We did not find an increase in success associated with the use of EMLA, but the number of cases in which EMLA was used was small. However, based on previous studies and the known benefits of procedural pain reduction, we would tentatively recommend the use of EMLA for LP.

In previous studies, the Cincinnati method of early stylet removal for LP was associated with increased success rates. ${ }^{[3,5]}$ In our study success appeared to be associated with late removal, but the number using early removal was small. The reason for the difference in results is unclear. Early stylet removal is not commonly taught in SA, so doctors may not be aware of the different methods and the risks and benefits associated with each. Larger studies are needed to determine which of the methods reduce traumatic taps, as well as the reasons why.

\section{Study limitations}

The study had several limitations, including those inherent to audits of current practice. It was a small study, there may have been sampling bias, and there was no randomisation of patients, no use of control groups and no observers of the procedures. Patients received sedation or not according to physician preference or normal practice, which is known to vary and may depend on the availability of monitoring in different areas of the hospital. There may have been recall bias as well as reporting bias because the questionnaires were completed after the LP result was known. Some problems were noted with the questionnaire, one question was often not completed, possibly owing to positioning on the page, and some questions overlapped. As this was not an efficacy study, it was not possible to quantify the risk reduction in absolute terms. For many of the factors examined the numbers in one group were small, so differences may have been missed. The depth of sedation achieved was not assessed and the numbers with each drug at each dose were small, so it was not possible to identify the most effective sedation regimen.

\section{Study implications}

This study suggests that the use of sedation is associated with an increase in the success of LP and provides some evidence towards the implementation and further evaluation of protocolised sedation. Further study is needed to determine the ideal medication and the optimal doses, and to assess the effectiveness of sedation in reducing the pain and trauma experienced by the child. Implementation of the use of sedation would require a change in organisation of the unit, with the drugs, monitoring, staff and appropriate environment readily available. This may be a challenge in resource-limited settings. Whether a protocol would then change practice, and whether this would increase success rates, will require further audit. Larger studies are also needed to determine the influence of other procedural factors and whether these can be modified to reduce the rate of unsuccessful LP. As this study was conducted in the medical emergency unit of a children's hospital, the results may not be generalisable to other healthcare facilities dealing with mixed patient loads, but nevertheless highlight the importance of providing procedural analgesia and sedation.

\section{Conclusions}

We found that procedural sedation was not routinely used but that it appeared to be associated with a reduction in unsuccessful LPs. The study also revealed a high prevalence of unsuccessful LPs in our institution, with the highest proportion being in infants aged $<1$ year. Whether a procedural sedation protocol in the MEU would reduce the rate of traumatic LPs, and hence unnecessary treatment and costs, requires further study.

Acknowledgements. We thank the RCWMCH staff and patients and the Faculty of Health Sciences, University of Cape Town.

Funding and financial disclosure. No funding was secured for this study, and the authors have no financial relationships relevant to this article to disclose.

\footnotetext{
1. Liu L, Johnson H, Cousens S, et al. Global, regional, and national causes of child mortality: An updated systematic analysis for 2010 with time trends since 2000. Lancet 2012;379(9832);2151-2161. http:// dx.doi.org/10.1016/S0140-6736(12)60560-1

2. Scarborough $\mathrm{M}$, Thwaites $\mathrm{G}$. The diagnosis and management of acute bacterial meningitis in resourcepoor settings. Lancet Neurology 2008;7(7):637-648. http://dx.doi.org/10.1016/S1474-4422(08)70139-X Nigrovic LE, Kuppermann N, Neuman MI. Risk factors for traumatic or unsuccessful lumbar punctures in children. Ann Emerg Med 2007:49(6):762-771. http://dx.doi.org/10.1016/j punctures in children.

4. Howard S, Gajiar A, Cheng C, Kritchevsky S. Risk factors for traumatic and bloody lumbar puncture in children with acute lymphoblastic leukemia. JAMA 2002;288(16):2001-2007. http://dx.doi. org/10.1001/jama.288.16.2001 org $/ 10.1001 /$ jama.288.16.2001

Baxter AL. Local anesthetic and stylet styles: Factors associated with resident lumbar puncture success. Pediatrics 2006;117(3):876-881. http://dx.doi.org/10.1542/peds.2005-0519

6. Zaoutis TE, Goyal M, Chu JH, et al. Risk factors for and outcomes of bloodstream infection caused by extended-spectrum $\beta$-lactamase-producing Escherichia coli and Klebsiella species in children. Pediatrics 2005;115(4):942-949. http://dx.doi.org/10.1542/peds.2004-1289

7. Thompson ML, Myers JE, Kriebel D. Prevalence odds ratio or prevalence ratio in the analysis of cross sectional data: What is to be done? Occup Environ Med 1998;55(4):272-277. http://dx.doi.org/10.1136/ oem.55.4.272

8. Glatstein MM, Zucker-Toledano M, Arik A, et al. Incidence of traumatic lumbar puncture: Experience of a large, tertiary care pediatric hospital. Clin Pediatr 2011;50(11):1005-1009. http://dx.doi. org/10.1177/0009922811410309

9. Reed A, Thomas J, Roelofse J, et al. Guidelines for the safe use of procedural sedation and analgesia for diagnostic and therapeutic procedures in children: 2010. S Afr J Anaesth Analg 2010;16(5):S1-S37.

0. Nigrovic LE, McQueen AA, Neuman MI. Lumbar puncture success rate is not influenced by familyNigrovic LE, McQueen AA, Neuman MI. Lumbar puncture success rate is not influenced by fa
member presence. Pediatrics 2007;120(4):e777-e782. http://dx.doi.org/10.1542/peds.2006-3442

11. Halperin D, Koren G, Attias D, et al. Topical skin anesthesia for venous, subcutaneous drug reservoir
. Halperin D, Koren G, Attias D, et al. Topical skin anesthesia for ven
and lumbar punctures in children. Pediatrics 1989;84(2):281-284.

12. Kaur G, Gupta P, Kuma A. A randomized trial of eutectic mixture of local anesthetics during lumbar puncture in newborns. Arch Pediatr Adolesc Med 2003;157(11):1065-1070. http://doi.org/10.1001/ archpedi.157.11.1065

13. Pinheiro JM, Furdon S, Ochoa LF. Role of local anesthesia during lumbar puncture in neonates. Pediatrics 1993;91(2):379-382
} 
Appendix 1. Lumbar puncture audit, February 2013

Lumbar puncture audit, February 2013

Date:

Time:

Patient weight: $\mathrm{kg}$

1. Are you a:

Senior registrar

Registrar
Medical officer/COSMO/SHO

Intern

2. How many LPs have you done?
$0-5$
$11-50$
$6-10$
$51+$

3. Indication for LP:

Exclude meningitis $\quad$ Reduce intracranial pressure
Measure opening pressure
Other (specify)

4. Needle used:
Black spinal (22G)
Other spinal (specify)
Other needle (specify)

5. Family member present?

Yes

No

6. Person holding child:

$\begin{array}{ll}\text { Student nurse } & \text { Staff nurse } \\ \text { Sister } & \text { Medical student } \\ \text { Doctor } & \text { Other }\end{array}$

7. Sedation and analgesia used - please tick and state dose:

None

EMLA

Lignocaine mL

Chloral hydrate mg

Ketamine IV__

Ketamine IM $\mathrm{mg}$

Midazolam mg

Morphine mg

Other (specify drug, dose and route) 
8. Vital sign monitoring during the procedure? (saturation and heart rate)

Yes

No

9. Was the patient moving?

Not at all

A little but did not affect the procedure

A lot - difficult procedure

10. When did you remove stylet?

When through skin/sub-cut tissue

11. Number of attempts required (i.e. number of times needle penetrated skin):

12. LP result
Dry tap
Bloody tap - frank blood
Bloodstained CSF
CSF obtained and sent to lab

\section{For completion by researcher:}

LP result

Appearance

Polys

Lymphs Erythrocytes

Glucose Protein

Gram stain Culture

Post tap headache

Yes

Treatment

Outcome

$\begin{array}{ll}\text { Discharged } & \text { Treated for meningitis }- \text { length stay __ days } \\ \text { Transfer to __ } & \text { Other complication }\end{array}$

